http://jmscr.igmpublication.org/home/ ISSN (e)-2347-176x ISSN (p) 2455-0450 crossref DOI: https://dx.doi.org/10.18535/jmscr/v7i8.127

Journal Of Medical Science And Clinical Research

\title{
Immunohistochemical Expression of WT1 in Glial Neoplasm and its Significance
}

\author{
Authors \\ Mathur Kusum ${ }^{1}$, Vijay Ramita ${ }^{2 *}$ \\ ${ }^{1}$ SeniorProfessor, ${ }^{2}$ Postgraduate Student \\ Department of Pathology, SMS Medical College, Jaipur, Rajasthan, India \\ Corresponding Author \\ Vijay Ramita \\ Postgraduate Student, Department of Pathology, SMS Medical College, Jaipur, Rajasthan, India
}

\begin{abstract}
Introduction: WT1 is a transcription factor related to growth and differentiation of cells. WT1 overexpression is observed in majority of primary glial tumours. However, it is differential expression of WT1 in different grades of tumour, that might influence the biological behavior of gliomas and thus prognosis. At present, as WT1 is regarded as a molecular target for immunotherapy for malignant gliomas, an appropriate evaluation of WT1 expression is essential. The present study was planned to assess the WT1 expression in various grades and types of glial neoplasm and correlate this with mean mitosis and tumour grade so as to exclude or include WT1 as a potential prognostic factor in patients with glial tumours.

Materials and Methods: A descriptive observational study was carried out in Department of Pathology, S.M.S. Medical College, Jaipur (Rajasthan). Total 50 cases of glioma were included in the study.These were stained with Hematoxylin and Eosin followed by Immunostaining with WT1. Clinical, histopathological and Immunohistochemical profile of glial tumours were recorded and analysed.

Results: Mean age of study subjects was 35.64 \pm 16.03 years (male 38.65 \pm 15.06 years and female $30.74 \pm 16.74$ years). $62 \%$ were males and $38 \%$ were females. Glioblastoma was the most frequent neoplasm (40\%). Highest number of Tumours belonged to Grade-IV (40\%) followed by Grade-II (38\%), Grade-I (12\%) and Grade-III (10\%) according to WHO grading system for glial tumours. Highest mean mitotic value was obtained in grade-IV tumours (7.80) while it was 0 in grade-I tumours $(p<0.001)$. Positive staining for WT 1 immunoexpression was observed in 45 cases (90\%) ranging from score 2 to score 6. Mean WT1 score for grade-I tumours was 2.50, for grade-II tumours 2.95, for grade-III tumours 2.60 and for grade-IV tumours 5.60 ( $p<0.001$ ). There was a strong correlation between WHO grade, mitosis and WT1 Score. Also, WT1 showed a direct independent correlation with mitotic activity of tumours.

Conclusion: WT1 immuno-expression bears a direct relation to tumour grade as well as mitotic activity. Thus, it appears to be involved in tumour growth and bears a prognostic significance. This makes it a useful molecular target for immunotherapy.

Keywords: Glial Neoplasm, Histopathology, Immunohistochemical, WT1 immunoexpression.
\end{abstract}

\section{Introduction}

The incidence of CNS tumours in India ranges from 5 to 10 per $1,00,000$ population with a rising trend. Astrocytomas $(38.7 \%)$ are the most common primary brain tumours with majority being high grade gliomas $(59.5 \%){ }^{1}$ The clinical 
behavior of Gliomas is determined by the grade of the tumor. The WHO classification 2016 divides gliomas in grade I, II, III and IV on the basis of morphology, invasiveness and resectability of tumor.

Majority of primary glial tumours and glioma cell lines show expression of WT1 while it is absent in normal as well as reactive astrocytes. ${ }^{2} \mathrm{WT} 1$ gene was first identified as a gene responsible for Wilm's tumour. It is located at chromosome $11 \mathrm{p} 13$ spans $50 \mathrm{~kb}$ genomic DNA, consists of 10 exons that transcript into mRNA of $3.2 \mathrm{~kb}$ size which in turns translates into a 429 amino acid protein $\mathrm{WT} 1{ }^{3}$ The alternative splicing of wt1 pre mRNA leads to four isoforms:WT1(-/-), WT1(/+), WT1(+/-), WT1(+/+). These isoforms are binding site specific, resulting in different effects on the expression of target genes. ${ }^{4}$

WT1 is a transcription factor that plays role in the normal development of mesodermally derived tissues like kidney, gonads, cardiac vasculature and in the proliferation of neuronal progenitors. ${ }^{5}$ However, wild type WT1 was found to be expressed in leukemia and other solid malignancies including gliomas. Being a transcription regulator, WT1 acts on many target genes involved in cellular growth., differentiation, proliferation and apoptosis. ${ }^{6}$ Transcription of several oncogenes is suppressed such as PDGF-A, TGF- $\beta$, EGFR and IGF1 receptor. While, expression of E-cadherin and proapoptotic proteins is enhanced. In this way WT1acts as a tumour suppressor gene. However, researches have shown that wild type WT1 has oncogenic properties. Upregulation of growth factor transcription and inactivation of p53 emphasize the tumour igenicity of WT1.

Recent researches have shown that growth of WT1 expressing tumour cells was inhibited by exposure to WT1 antisense oligomers, indicating that WT1can be used as a target molecule for immunotherapy in treatment of glial neoplasm. ${ }^{7}$ In this sequela, WT1 peptide vaccines were developed and successful phase I and phase II clinical trials were carried out for treatment of patients with recurrent glioblastoma. ${ }^{8,9}$ The efficacy of this immunotherapy proves WT1 to be an important cancer antigen of therapeutic value.

As the WT1 promotes proliferation and survival of cells, the biological behavior of glioma might be influenced by differential expression of WT1 in different grades of tumour. An appropriate evaluation of WT1 expression is essential at present and immunohistochemical analysis for WT1 expression in routinely processed histological sections is essential to judge the eligibility of glioma patients for WT1 targeted immunotherapy, adding to the survival.

\section{Materials and Methods}

It was a laboratory based, cross sectional, observational study, carried out in Department of Pathology, SMS Medical College, Jaipur (Rajasthan) from March 2017 to September 2018. Prior approval from institutional ethics committee was taken and all neurosurgical cases reported as glioma were included in the study while nonneoplastic lesions of CNS, non-glial neoplasm and autolysed tissue specimen were excluded. Total 50 cases were taken for the study.

Following adequate fixation for about 12-24 hours, the tissues were submitted for routine processing, following which the paraffin embedded serial sections of 3-4 micron thickness were obtained. These were stained with Hematoxylin and Eosin stain and Immunohistochemistry was applied afterwards. The clinical data was obtained from requisition form and specimens were microscopically examined for the type and grade of the tumours as well as for mitotic count. Immunohistochemical profile of glial tumours was studied by using WT1.

\section{Interpretation of WT1 Immunostaining}

All specimens were observed under light microscope. Only samples in which endothelial cells showed WT1 expressions were included in the study. Endothelial cells were utilized as internal positive control and normal glial tissue were utilized as internal negative control. WT1 
index was determined as the sum of WT1-positive relative frequency score and WT1 staining score. WT1 staining score was considered as:

0 (negative): no staining

1 (weak): mildly increased staining compared to the normal glial cells

2 (intermediate): staining at intensity between 1 and 3

3 (strong): significantly increased staining in tumour cells compared with normal glial cells.

Relative frequency of WT1-positive tumour cells was scored as $0(0 \%), 1(<25 \%), 2(25-75 \%)$ and $3(>75 \%)$.

In addition, the distribution of WTI protein expression was evaluated in the whole tissue section and also intra-cellular (cytoplasmic /nuclear).

\section{Statistical Analysis}

Data were entered in Microsoft Excel 2010 spreadsheets. Qualitative data were expressed in percentages while quantitative data were expressed as mean and standard deviation, ANOVA and Pearson's correlation coefficient was used to see association and correlation between WT1 expression, tumour grade and mitosis. P-value $<0.05$ was considered statistically significant.

\section{Results}

A total of 50 cases of glial neoplasm were studied for histomorphology and immunohistochemical expression of WT1. The age range of study subjects was 4 years -68 years. The highest numbers of cases were in the age group of 21-40 years $(46 \%)$.Out of 50 cases, 31 were males $(62 \%)$ and 19 were females $(38 \%)$.

Glioblastoma accounted for maximum number of cases constituting $40 \%$ followed by diffuse astrocytoma (20\%), oligodendroglioma (12\%) and pilocytic astrocytoma (10\%) (Figure-1). Tumours were graded using WHO grading system for glial tumours. 6 tumours were of WHO grade-I (12\%), 19 were of grade-II $(38 \%), 5$ cases were of gradeIII (10\%) and 20 cases belong to grade-IV (40\%). Grade-I glial tumours included pilocytic astrocytoma and subependymal giant cell astrocytoma (SEGA). Grade-II tumours were diffuse astrocytoma, oligodendroglioma, ependymoma and pleomorphic xanthoastrocytoma (PXA). Grade-III tumours were anaplastic astrocytoma, anaplastic oligodendroglioma and anaplastic ependymoma. Glioblastoma were grade-IV tumours.

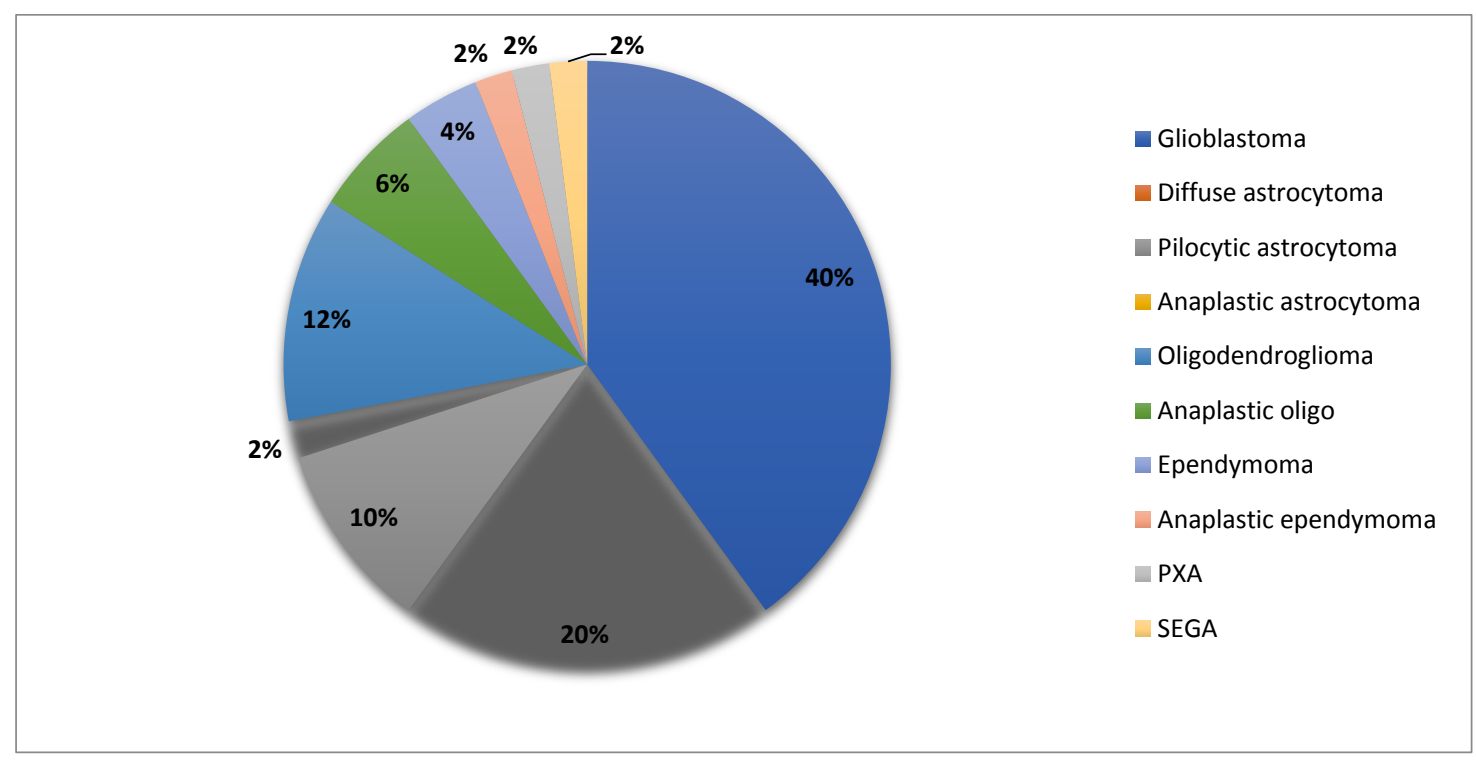

Figure -1: Distribution of type of tumours

Mitotic figures per 10 high power field (400X) were counted for each tumour and mean mitosis for each grade group was calculated. Highest value was obtained in grade-IV tumours (7.80) 
while it was 0 in grade-I tumours. Significant difference in the mitosis among different grades (p-value $<0.001)$ was observed. This confirmed the prognostic value of mitotic activity in gliomas (Table-1).

Table-1: Mean mitosis in different grade of glial tumours

\begin{tabular}{|c|c|c|c|c|c|}
\hline WHO Grade & $\mathbf{N}$ & Mean mitosis & SD & P-Value* & Significant difference from \\
\hline Grade-I & 6 & - & - & \multirow{5}{*}{$<0.001$} & - \\
\hline Grade-II & 19 & 0.21 & 0.42 & & III, IV \\
\hline Grade-III & 5 & 5.00 & 1.58 & & II, IV \\
\hline Grade-IV & 20 & 7.80 & 2.63 & & II, III \\
\hline Total & $\mathbf{5 0}$ & 3.70 & 4.05 & & - \\
\hline
\end{tabular}

Out of 50 cases, 45 cases (90\%) showed positive immunoexpression for WT1, ranging from score 2 to 6 . In all the positive cases WT 1 expression was cytoplasmic and maximum staining was observed in areas of high cellularity and high tumour vascularity. All astrocytic and ependymal tumours showed positive WT 1 immunostaining while out of 9 oligodendrocytictumours only 4 (3 grade II and 1 Grade IIITumours) showed WT 1 expression of mild intensity. The positive staining in oligodendrocytictumours were mainly due to WT1 expression by minigemistocytes and gliofibrillary oligodendrocytes. Mean WT1 score for grade-I tumours was 2.50 , for grade-II tumours 2.95 , for grade-III tumours 2.60 and for grade-IV tumours 5.60. This observation shows significant difference between mean WT1 score among different grades $(p<0.001)$ (Table-2).Regression analysis showed a strong correlation between WHO grade and WT1 Score (Figure-2).

Table-2: Mean WT1 Score in different grade of glial tumours

\begin{tabular}{|c|c|c|c|c|c|}
\hline WHO Grade & $\mathbf{N}$ & Mean WT1 Score & SD & P- Value ${ }^{*}$ & Significant difference from ${ }^{\#}$ \\
\hline Grade-I & 6 & 2.50 & 0.55 & \multirow{5}{*}{$<0.001$} & IV \\
\hline Grade-II & 19 & 2.95 & 1.54 & & IV \\
\hline Grade-III & 5 & 2.60 & 2.79 & & IV \\
\hline Grade-IV & 20 & 5.60 & 0.68 & & I,II,III \\
\hline Total & 50 & 3.92 & 1.91 & & \\
\hline
\end{tabular}

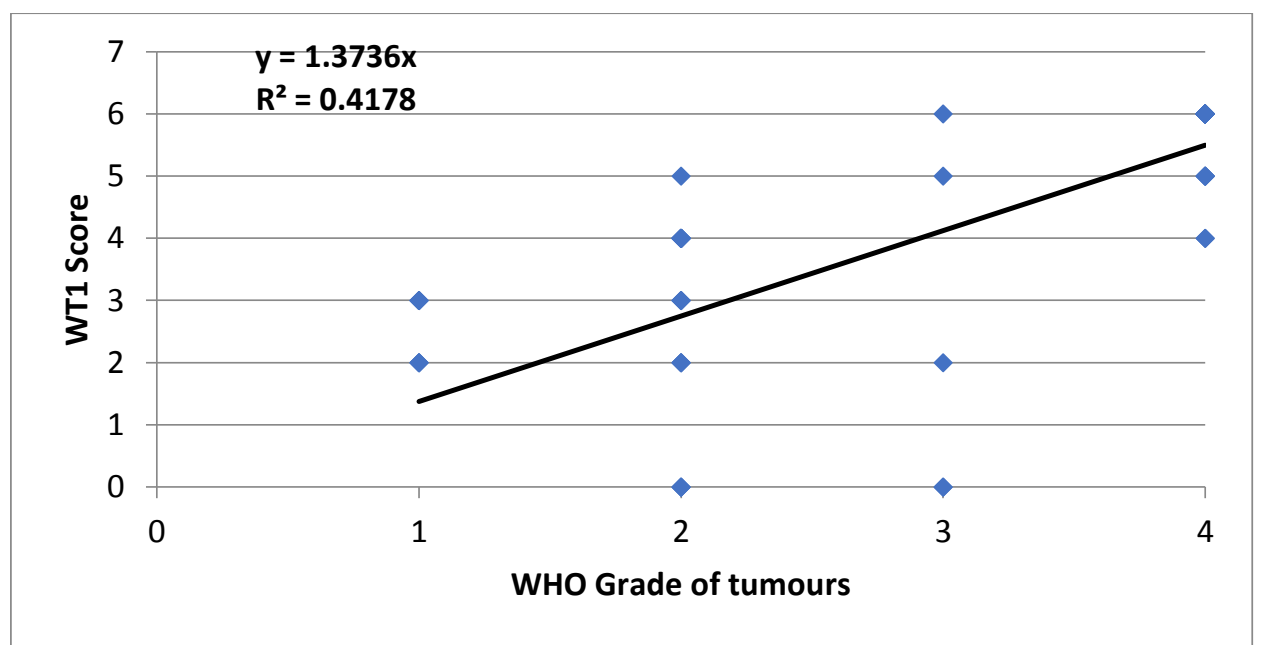

Figure-2: Scatter plots and regression lines showing the relationship between WHO Grade of tumours and WT1 Score 


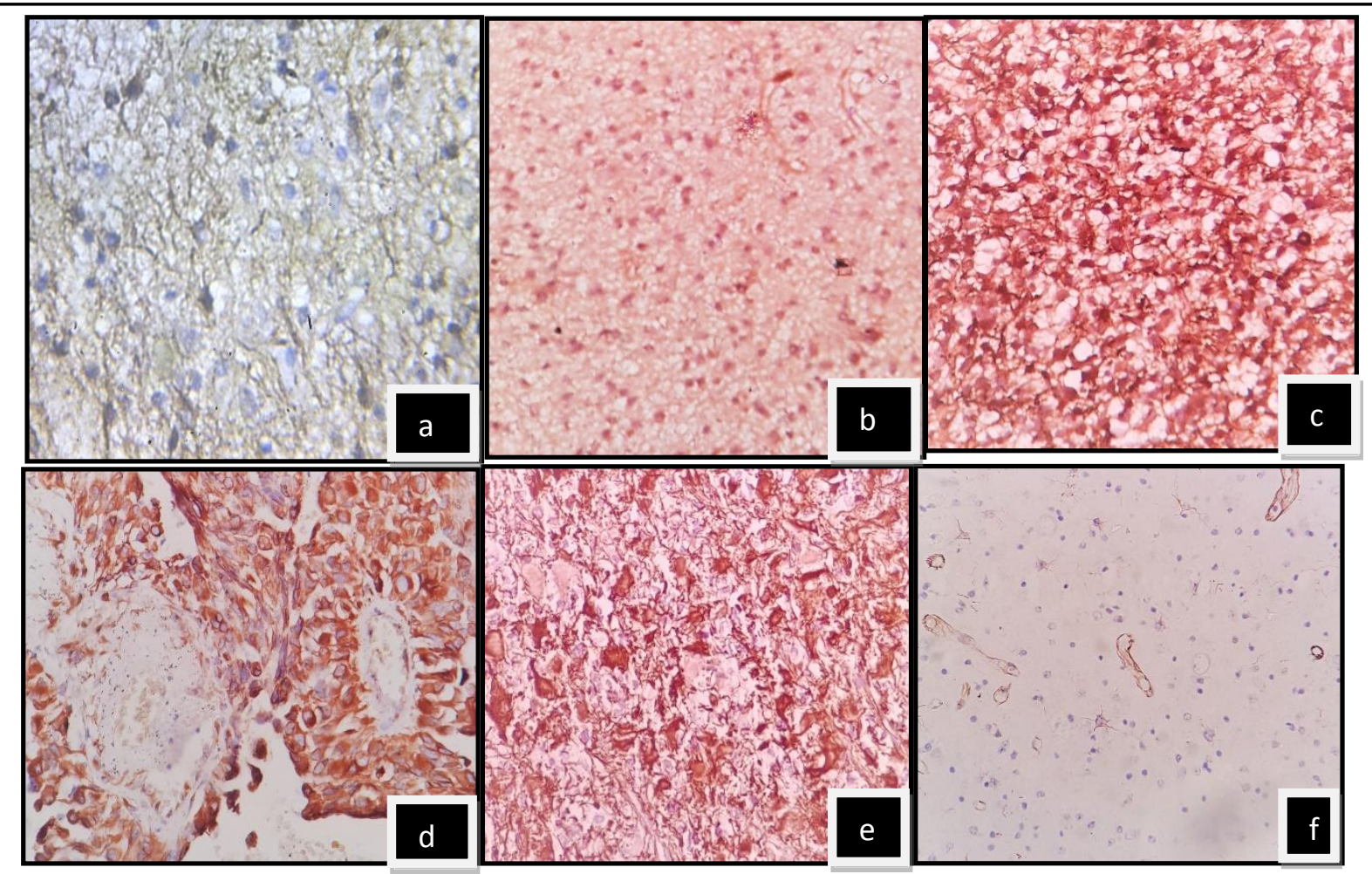

Figure 3 : WT1 expression in various grades of glioma- (a) WT1 score 2 in pilocytic astrocytoma (b) Score 4 in diffuse astrocytoma (c) Score 6 in anaplastic astrocytoma (d) Score 5 in anaplastic ependymoma (e) Score 6 in Glioblastoma (f) Negative expression in oligodendroglioma.

\section{Discussion}

Glial neoplasm are the most common brain tumours and the 'Glioblastoma' are the most aggressive tumours with worst prognosis and high recurrence. Glioma tumourigenesis has been found to be associated with overexpression of WT1 protein in neoplastic cells. In the present study, the possible prognostic value of WT1 expression in the glial tumours was evaluated.

In this study, the mean age of patients was 35.64 years which was similar to other studies by Bassam et $\mathrm{al}^{10}$ (34.85 years) and Ibrahim et $\mathrm{al}^{11}$ (32.2 years). $62 \%$ patients were males and $38 \%$ were females which was in concordance with the study by Ibrahim et al ${ }^{11}(66.25 \% \mathrm{v} / \mathrm{s} 33.75 \%)$ and Schittenhelm et $\mathrm{al}^{12}(62 \% \mathrm{v} / \mathrm{s} 38 \%)$. In present study maximum cases were Glioblastoma $(40 \%)$ that was close to a study by Bassam et $\mathrm{al}^{10}(38 \%)$ while Ibrahim $\mathrm{B}$ et $\mathrm{al}^{11}$ observed slightly lower $(27.5 \%)$ and Schittenhelm et $\mathrm{al}^{12}$ observed higher $(53.32 \%)$ proportion, however, glioblastomas were the most frequent gliomas.

On assessing the level of WT1 expression immunohistochemically, percentage of immune expression was $90 \%(44 / 50)$ of all cases which approached the study by Ibrahim et al ${ }^{11}(91.24 \%)$ and Hashiba et $\mathrm{al}^{13}(95.9 \%)$. The study by Oji et $\mathrm{al}^{14}(96 \%)$ observed positivity in almost all cases. WT1 expression in most of the cases was found to be localized to the cytoplasm of neoplastic cells. This was similar to study by Oji et $\mathrm{al}^{14}$ who detected WT1 protein in the cytoplasm of WT1 immunopositive tumour cells, suggesting a function of WT1 other than transcription regulation, possibly in RNAmetabolism.In all positive cases, maximum staining wasobserved in areas of high cellularity that is in harmony with Hashiba et $\mathrm{al}^{13}$ who found strong WT1 protein expression in the anaplastic portions, areas with high cellularity and perivascular proliferation. This points towards an important role of WT1 gene in glioma cell proliferation as well as its possible use as proliferation marker in glial tumours.

WT 1 expression level in astrocytomas was higher as compared to that in oligodendrocytomas similar 
to studies by Rauscher et al ${ }^{15}$ and Schittenhelm et $\mathrm{al}^{12}$. This suggests a role ofWT1 in astrocytic differentiation of glial cells. Thus, WT 1 can be used to differentiate astrocytic and oligodendrocytic tumours and is of a diagnostic utility.

In current study, significant difference between mean WT1 scores and different tumour grades was observed. This was in league with Hashiba et $\mathrm{al}^{13}$ who found, higher grade astrocytic tumour had higher WT1 expression score. Oji et al ${ }^{14}$ showed that high WT 1 protein expression level correlated with high grade of astrocytic tumour. Schittenhelm et $\mathrm{al}^{12}$ observed that $52 \%$ of diffuse astrocytoma showed positive WT 1 immunoexpression while high grade glioma showed $>75 \%$ cells positive for WT1 expression (score 6). Bassamet $\mathrm{al}^{10}$ observed higher scores in high grade tumours suggesting positive correlation. To conclude, WT 1 expression score increased with increasing tumour grade and can be considered as oncogene culprit for tumour genesis and important in prognosis.Apart from the tumour grade, WT1 score was also evaluated for a direct correlation with mitotic activity. The mean mitotic count of each WT1 score was found to have a significant difference. This was in accordance with Bassam et $\mathrm{al}^{10}$ who found a correlation close to significance. A strong positive correlation between WT1 Score and mitosis for all scores was found in that study. This infers an independent correlation between WT1 score and Mitotic activity, highlighting a proliferation promoting role ofWT1.

\section{Conclusion}

Significant proportion of glial tumours show WT1 immunoexpression. Its expression in astrocytic tumours is higher than in oligodendrocytic tumours. Thus, these may be used as diagnostic markers to differentiate between these two entities. WT1 is a suitable molecule for the targeted immunotherapy in patients with glioblastoma because it is highly expressed by the tumour cells. The treatment of recurrent high grade glioma will be revolutionized with the introduction of WT1 targeted therapy. Thus, for the sake of better patient management, WT1 immunoexpression level should be evaluated for cases of glial tumourin routine practice.

\section{References}

1. Dasgupta A, Gandhi T, Jalali R. Indian data on central nervous tumours: A summary of published work. Southa Asian J Cancer. 2016;5(3):147-53.

2. Schittenhelm J, Mittelbronn M, Nguyen TD, Meyermann R, Beschorner R. WT1 expression distinguish esastrocytic tumour cells from normal and reactive astrocytes. Brain Pathol 2008;18(3):344-53.

3. Gessler M, Poustka A, Cavenee W, Neve RL, Orkin SH, Bruns GAP. Homozygous deletion in Wilms tumours of a zinc finger gene identified by chromosome jumping. Nature1990;343:774-8.

4. Sharma, PM, M Bowman, SL Madden, FJ Rauscher and S Sukumar. RNA editing in the Wilms' tumour susceptibility gene, WT1. Genes Dev. 1994;8:720-31.

5. Davies R, Moore A, Schedl A, Bratt E, Miyahawa K, Ladomery Metal. Multiple roles for the Wilms' tumour suppressor, WT1. Cancer Res 1999;59:1747-50

6. Sugiyama H. Wilms' tumour gene WT1: its oncogenic function and clinical application. Int J Hematol2001;73:177-87.

7. Sugiyama H. Cancer immunotherapy targeting WT1 protein. Int J Hematol2002;76:127-32.

8. S Izumoto, A Tsuboi, Y Oka, T Suzuki, T Hashiba, N kagwa et al. Phase II clinical trial of Wilms' tumour 1 peptide vaccination for patients with recurrent glioblastoma multiforme. J. Neurosurg 2008;108:963-71.

9. N Hashimoto, A Tsuboi, N Kagwa, Y Chiba, SIzumoto, M Kinoshitaetal. Wilms tumour 1 peptide combined with temozolamide against newly diagnosed 
Glioblastoma: safety and impact on immunological response. Cancer Immunol. Immunother 2015;64:707-16

10. Bassam AM, Lubna O, Abdel-Salam and D Khairy. WT1 expression in Glial tumours: Its Possible Role in Angiogenesis and Prognosis. Academic J CancerRes. 2014;7(2):50-58.

11. Ibrahim BB, MM Sami Salem, AAM Hareedy, NSAl Hariry. Histopathological study and Immunohistochemical Expression of WT1 Protein in Different Types and Grades of Astrocytic Tumours. Academic J Cancer Res. 2016;9(1):01-06.

12. Schittenhelm J, Beschorner R, Simon P, Tabatabai G, Herrmann C, Schlaszus H, Capper D et al. Diagnostic value of WT1 in neuroepithelial tumours. Neuropathol Appl Neurobiol 2009;35(1):69- 81.

13. Hashiba T, $\mathrm{S}$ Izumoto, $\mathrm{N}$ Kagwa, $\mathrm{T}$ Suzuki, N Hashimoto, M Maruno, T Yoshimine. Expression of WT1 protein and correlation with cellular proliferation in glial tumours. Neurol Med Chir (Tokyo).2007;47:165-70.

14. Oji Y, T Suzuki, Y Nakano, M Maruno, S Nakatsuka, T Jomgeowetal. Over expression of the Wilms' tumour gene WT1 in astrocytic tumours. Cancer Sci. 2004;95:822-7.

15. Raucher J, R Beschorner, M Gierke, S Bisdas, C Braun, FH Ebner, J Schittenhelm. WT1 expression increases with malignancy and indicates unfavourable outcome in astrocytoma. J. Clin Pathol 2014;67(7):556-61. 